\title{
Harris Extended Length Biased Exponential Distribution and its Applications
}

\section{Jismi Mathew}

Department of Statistics, Vimala College(Autonomous), Thrissur, India-680009

\begin{tabular}{ll}
\hline ARTICLE INFO & ABSTRACT \\
\hline $\begin{array}{l}\text { Published Online: } \\
\text { 15 April 2020 }\end{array}$ & $\begin{array}{l}\text { In this article, a generalization of the Length biased Exponential distribution called Harris } \\
\text { extended Length biased Exponential distribution is studied, and its properties are discussed. The } \\
\text { parameters of the proposed model are estimated by using maximum likelihood estimation } \\
\text { method.The new distribution is applied to the real data sets to establish the flexibility of the } \\
\text { newly developed model. }\end{array}$ \\
$\begin{array}{l}\text { Corresponding Author: } \\
\text { Jismi Mathew }\end{array}$ & \begin{tabular}{l} 
new \\
\hline
\end{tabular}
\end{tabular}

KEYWORDS: Length Biased Exponential distribution, Harris Generalization, maximum likelihood estimation, hazard functions, Order statistics

\section{Introduction}

Addition of new parameters to extend classical distributions in order to get more flexible families of distribution has been investigated by several authors in the existing literature. In many applied areas such as lifetime analysis, finance and insurance, there is a clear need for extended forms of the classical distributions. Lifetime processes have recently received several attentions through the modelling of their distribution. Recent developments aims to focus on new techniques for building meaningful distributions.

Dara and Ahmad (2012) introduced the Length-Biased Exponential (LBE) distribution and they proved that the LBE is more flexible than the exponential distribution with cdf

$$
G(x)=1-\left(\frac{x}{\eta}+1\right) e^{-\frac{x}{\eta}} ; x>0, \eta>0
$$

and the pdf is

$$
g(x)=\frac{x e^{-\frac{x}{\eta}}}{\eta^{2}} ; x>0, \eta>0
$$

The hazard rate function of the LBE distribution is

$$
h(x)=\frac{x e^{-\frac{x}{\eta}}}{\eta^{2}\left(\frac{x}{\eta}+1\right) e^{-\frac{x}{\eta}}} ; x>0, \eta>0
$$

Recently Muhammad et al.(2017), Obubu Maxwell et al. (2019) studied various forms of LBE models.

Aly and Benkherouf (2011) introduced a new family of distributions, called Harris Extended (HE) family by adding two new parameters to a baseline distribution. Since the method is based on the probability generating function introduced by Harris (1948), the resulting family of distributions is known as Harris Extended (HE) family of distributions. If $\mathrm{g}(\mathrm{x}), \bar{G}(x)$ and $h_{G}(x)$ denote the probability density function (pdf), survival function (sf) and hazard rate function (hrf) of a parent distribution and the parameters $\delta$ and $\mathrm{k}$ are additional shape parameters, then the sf of $\mathrm{HE}$ family of distributions is given by

$\bar{F}(x)=\left[\frac{\delta(\bar{G}(x))^{k}}{1-\bar{\delta} \bar{G}(x)^{k}}\right]^{\frac{1}{k}} ; x>0, \delta>0, \bar{\delta}=1-\delta, k>0$

The HE density function is

$f(x)=\frac{\delta^{\frac{1}{k}} g(x)}{\left[1-\bar{\delta} \bar{G}(x)^{k}\right]^{1+\frac{1}{k}}} ; x>0, \delta>0, \bar{\delta}=1-\delta, k>0$

The hrf of the HE distribution is given by

$$
h(x)=\frac{h_{G}(x)}{\left[1-\bar{\delta} \bar{G}(x)^{k}\right]} ; x>0
$$

where $h_{G}(x)$ denotes the hazard rate function of the baseline distribution. Batsidis and Lemonte (2014) considered the HE family of distributions with respect to some lifetime models. Recently, Jose and Varghese (2019), Jose and Remya (2014), Pinho et al. (2015), Jose and Paul (2018),Gauss et al.(2019),Jose et al.(2018) studied various forms of HE models. Thus, we proposed a new generalization of the length biased exponential distribution called the Harris extended length biased exponential (HELBE) distribution for modeling lifetime data. In the rest of the paper, we define the HELBE densities, the survival and hazard functions, and plots for different parameter values in Section 2; some of the statistical properties of the proposed HELBE distribution are discussed in section 3, Application of the HELBE distribution to lifetime data in section 4 . The concluding remark is presented in section 5 . 


\section{Harris Extended Length biased Exponential distribution}

Substituting the sf of LBE distribution in (4), we get the sf of Harris Extended Length biased Exponential (HELBE) distribution. Then the one parameter LBE distribution tends to a three parameter HELBE. The Survival function of the HELBE distribution is given by,

$$
\bar{F}(x)=\left(\frac{\delta\left(\frac{x}{\eta}+1\right)^{k} e^{-\frac{k x}{\eta}}}{1-\bar{\delta}\left(\frac{x}{\eta}+1\right)^{k} e^{-\frac{k x}{\eta}}}\right)^{1 / k} ; x>0, \eta>0, k>0, \delta>
$$

$0, \bar{\delta}=1-\delta$

Then the pdf is

$$
f(x)=\frac{x \delta^{1 / k} e^{-\frac{x}{\eta}}\left(1-\bar{\delta}\left(\frac{x}{\eta}+1\right)^{k} e^{-\frac{k x}{\eta}}\right)^{-\frac{1}{k}-1}}{\eta^{2}} ; x>0, \eta>0, k>
$$

$0, \delta>0, \bar{\delta}=1-\delta$

where $\eta$ is a scale parameter representing the characteristic life and $\delta$ and $\mathrm{k}$ are the shape parameters of HELBE distribution. A random variable $\mathrm{X}$ following Harris Extended Length biased Exponential distribution is denoted by $\mathrm{X}$ $\operatorname{HELBE}(\mathrm{x}, \eta, \delta, \mathrm{k})$.

The cumulative distribution function is

$$
F(x)=1-\bar{F}(x)=1-\left(\frac{\delta\left(\frac{x}{\eta}+1\right)^{k} e^{-\frac{k x}{\eta}}}{1-\bar{\delta}\left(\frac{x}{\eta}+1\right)^{k} e^{-\frac{k x}{\eta}}}\right)^{1 / k} ; x>0, \eta>
$$

$0, k>0, \delta>0, \bar{\delta}=1-\delta$

The hazard function is

$$
h(x)=\frac{x}{\eta^{2}\left(1+\frac{x}{\eta}\right)\left(1-\bar{\delta}\left(\frac{x}{\eta}+1\right)^{k} e^{-\frac{k x}{\eta}}\right)}
$$

The HELBE distribution contains the following well-known distributions:

(i)Length biased Exponential (LBE) Distribution. For $\delta=1$ and $\mathrm{k}=0$, the HELBE distribution reduces to

$$
f(x)=\frac{x e^{-\frac{x}{\eta}}}{\eta^{2}} ; x>0, \eta>0
$$

which is the density of the LBE distribution introduced by Dara and Ahmad (2012).

(ii) Marshall Olkin Length Biased Exponential (MOLBE) Distribution. For $\mathrm{k}=1$, the HELBE distribution reduces to

$$
f(x)=\frac{x \delta e^{-\frac{x}{\eta}\left(1-(1-\delta)\left(\frac{x}{\eta}+1\right) e^{-\frac{x}{\eta}}\right)^{-2}}}{\eta^{2}} ; x>0, \eta>0, \delta>0
$$

which is the density of the MOLBE distribution introduced by Muhammad et al.(2017).

Figs. 1-3 shows some possible shapes of the pdf and hazard function of the HELBE distribution for selected values of parameters.
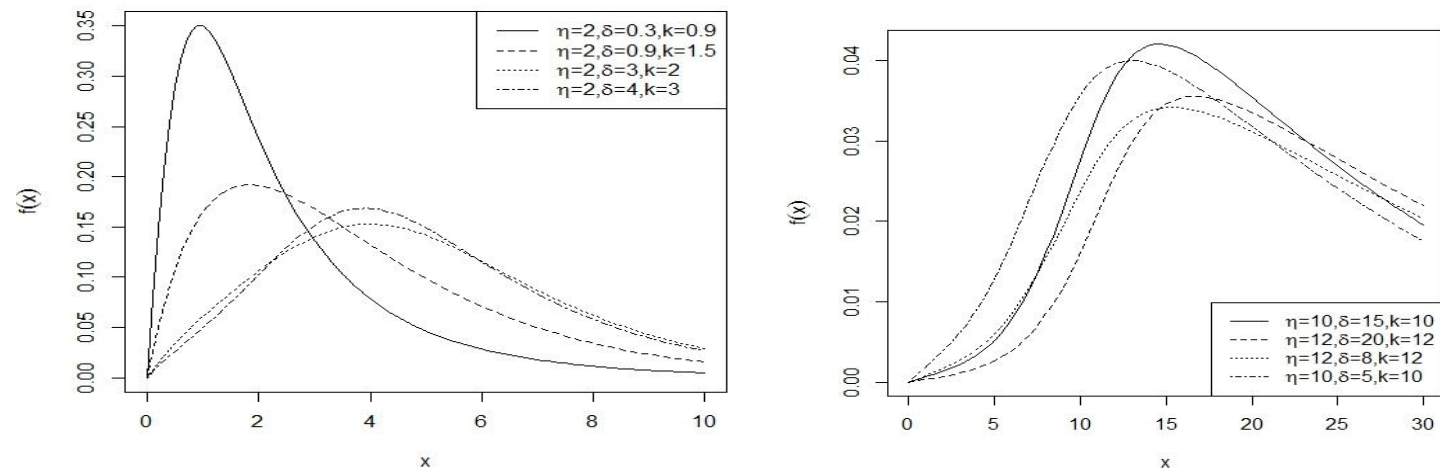

Fig 1: Graph for HELBE density function at different parameter values
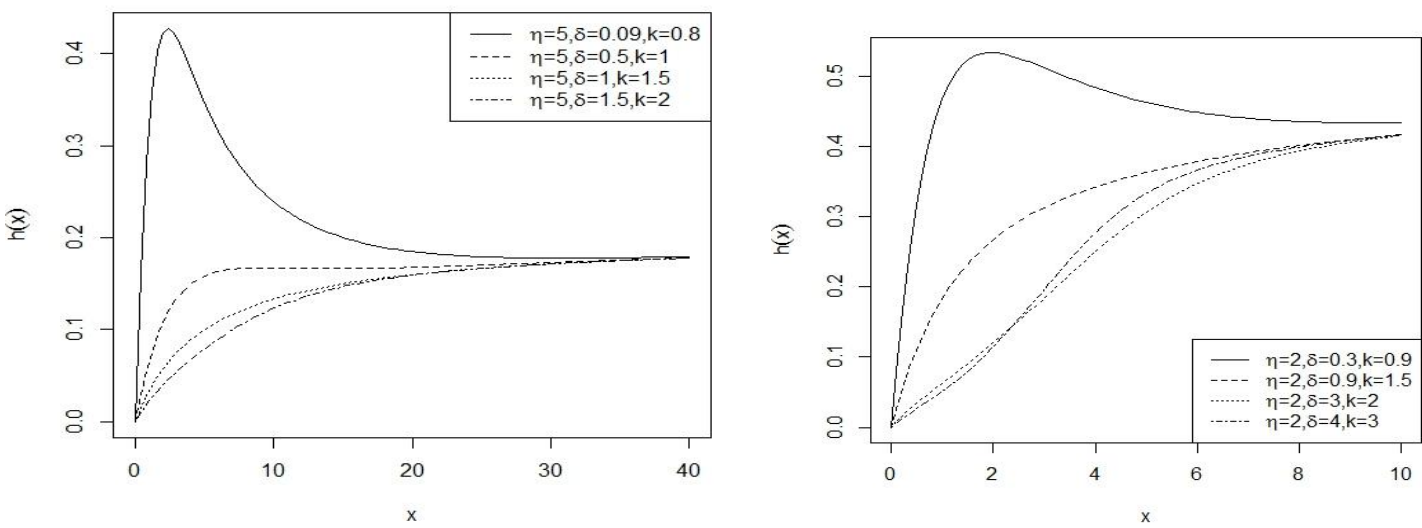

Fig 2: Graph for hazard rate function at different parameter values. 

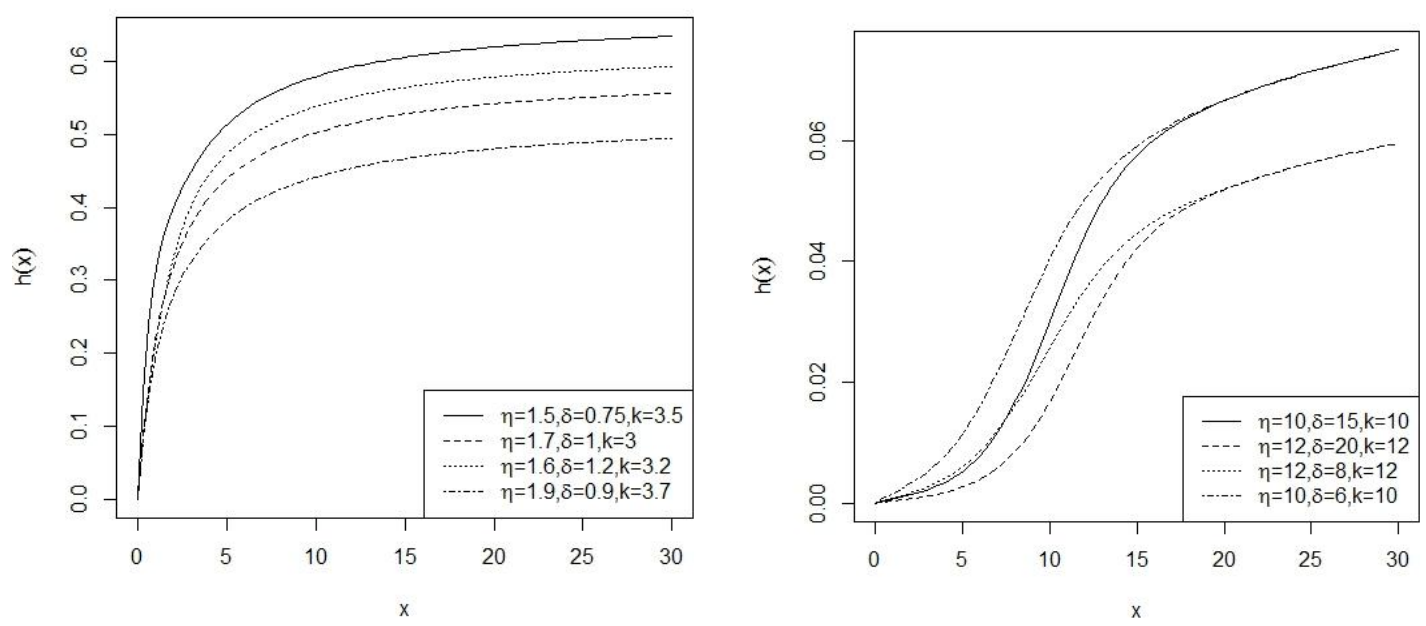

Fig 3: Graph for hazard rate function at different parameter values.

\section{Statistical Properties of the HELBE Distribution}

In this section, we study some statistical properties of HELBE distribution like asymptotic behavior, parameter estimation and order statistics.

\subsection{Asymptotic Behavior}

The behavior of the HELBE model is checked as $x \rightarrow 0$ and as $x \rightarrow \infty$

$$
\begin{aligned}
& \lim _{x \rightarrow 0} f(x)=\lim _{x \rightarrow 0} \frac{x \delta^{1 / k} e^{-\frac{x}{\eta}}\left(1-(1-\delta)\left(\frac{x}{\eta}+1\right)^{k} e^{\left.-\frac{k x}{\eta}\right)^{-\frac{1}{k}-1}}\right.}{\eta^{2}}=0 \\
& \lim _{x \rightarrow \infty} f(x)=\lim _{x \rightarrow \infty} \frac{x \delta^{1 / k} e^{-\frac{x}{\eta}}\left(1-(1-\delta)\left(\frac{x}{\eta}+1\right)^{k} e^{-\frac{k x}{\eta}}\right)^{-\frac{1}{k}-1}}{\eta^{2}}=0
\end{aligned}
$$

This shows that the HELBE distribution is unimodal. The pdf plot in the above figure also shows the HELBE model has only one peak. This support that the HELBE distribution has only one mode. We can also show that, $\lim _{x \rightarrow \infty} F(x)=1$

$$
\lim _{x \rightarrow \infty} F(x)=\lim _{x \rightarrow \infty}\left\{1-\left(\frac{\delta\left(\frac{x}{\eta}+1\right)^{k} e^{-\frac{k x}{\eta}}}{1-(1-\delta)\left(\frac{x}{\eta}+1\right)^{k} e^{-\frac{k x}{\eta}}}\right)^{1 / k}\right\}=\{1-0\}^{1 / k}=1
$$

\subsection{Maximum Likelihood Estimation}

The maximum likelihood estimation (MLE) method is used for the parameter estimation of HELBE distribution. Let $X_{1}, X_{2} \ldots X_{n}$ be a random sample from Harris Extended Length Biased Exponential (HELBE) distribution. Then the sample's likelihood function is given by

$$
L=\prod_{i=1}^{n} \frac{\delta^{1 / k} x_{i} e^{-\frac{x_{i}}{\eta}}\left(1-(1-\delta)\left(\frac{x_{i}}{\eta}+1\right)^{k} e^{-\frac{k x_{i}}{\eta}}\right)^{-\frac{1}{k}-1}}{\eta^{2}}
$$

The log-likelihood function reduces to,

$$
\begin{aligned}
& \log L= \\
& \frac{-\eta(k+1) \sum_{i=1}^{n} \log \left(1-(1-\delta)\left(\frac{x_{i}}{\eta}+1\right)^{k} e^{-\frac{k x_{i}}{\eta}}\right)+\eta k \sum_{i=1}^{n} \log \left(x_{i}\right)-k \log (e) \sum_{i=1}^{n} x_{i}+\eta n \log (\delta)-2 \eta k n \log (\eta)}{\eta k}
\end{aligned}
$$


The partial derivatives of this log-likelihood function is given by

$$
\begin{aligned}
& \frac{\frac{(1-\delta) k x_{i}\left(\frac{x_{i}}{\eta}+1\right)^{k-1} e^{-\frac{k x_{i}}{\eta}}}{\eta^{2}}-\frac{(1-\delta) k \log (e) x_{i}\left(\frac{x_{i}}{\eta}+1\right)^{k} e^{-\frac{k x_{i}}{\eta}}}{\eta^{2}}}{\eta-(1-\delta)\left(\frac{x_{i}}{\eta}+1\right)^{k} e^{-\frac{k x_{i}}{\eta}}} \\
& -\frac{(k+1) \sum_{i=1}^{n} \log \left(1-(1-\delta)\left(\frac{x_{i}}{\eta}+1\right)^{k} e^{-\frac{k x_{i}}{\eta}}\right)+k \sum_{i=1}^{n} \log \left(x_{i}\right)-2 k n \log (\eta)+n \log (\delta)-2 k n}{\eta k} \\
& -\frac{-\eta(k+1) \sum_{i=1}^{n} \log \left(1-(1-\delta)\left(\frac{x_{i}}{\eta}+1\right)^{k} e^{-\frac{k x_{i}}{\eta}}\right)+\eta k \sum_{i=1}^{n} \log \left(x_{i}\right)}{\eta^{2} k} \\
& -\frac{k \log (e) \sum_{i=1}^{n} x_{i}+\eta n \log (\delta)-2 \eta k n \log (\eta)}{\eta^{2} k} \\
& \frac{\partial \log \mathrm{L}}{\partial \delta}=\frac{\frac{\eta n}{\delta}-\eta(k+1) \sum_{i=1}^{n} \frac{\left(\frac{x_{i}}{\eta}+1\right)^{k} e^{-\frac{k x_{i}}{\eta}}}{1-(1-\delta)\left(\frac{x_{i}}{\eta}+1\right)^{k} e^{-\frac{k x_{i}}{\eta}}}}{\eta k} \\
& =\frac{-\eta(k+1) \sum_{i=1}^{n} \frac{\frac{(1-\delta) \log (e) x_{i}\left(\frac{x_{i}}{\eta}+1\right)^{k} e^{-\frac{k x_{i}}{\eta}}}{\eta}-(1-\delta)\left(\frac{x_{i}}{\eta}+1\right)^{k} \log \left(\frac{x_{i}}{\eta}+1\right) e^{-\frac{k x_{i}}{\eta}}}{1-(1-\delta)\left(\frac{x_{i}}{\eta}+1\right)^{k} e^{-\frac{c x_{i}}{\eta}}}}{\eta k} \\
& -\frac{\eta \sum_{i=1}^{n} \log \left(1-(1-\delta)\left(\frac{x_{i}}{\eta}+1\right)^{k} e^{-\frac{k x_{i}}{\eta}}\right)+\eta \sum_{i=1}^{n} \log \left(x_{i}\right)-\log (e) \sum_{i=1}^{n} x_{i}-2 \eta n \log (\eta)}{\eta k} \\
& -\frac{-\eta(k+1) \sum_{i=1}^{n} \log \left(1-(1-\delta)\left(\frac{x_{i}}{\eta}+1\right)^{k} e^{-\frac{k x_{i}}{\eta}}\right)+\eta k \sum_{i=1}^{n} \log \left(x_{i}\right)}{\eta k^{2}} \\
& -\frac{k \log (e) \sum_{i=1}^{n} x_{i}+\eta n \log (\delta)-2 \eta k n \log (\eta)}{\eta k^{2}}
\end{aligned}
$$

The maximum likelihood estimator $(\hat{\eta}, \hat{\delta}, \hat{k})$ of the parameters $(\eta, \delta, k)$ can be obtained by solving the equations $\frac{\partial \operatorname{logL}}{\partial \eta}=0$, $\frac{\partial \log \mathrm{L}}{\partial \delta}=0$ and $\frac{\partial \log \mathrm{L}}{\partial k}=0$. This solution can also be obtained by using R software with $n l m$ package .

\subsection{Order Statistics}

Let $X_{1}, X_{2} \ldots X_{n}$ be a random sample of size $\mathrm{n}$ from the HELBE distribution and $X_{1: n}, X_{2: n}, \ldots, X_{n: n}$ are the corresponding order statistics, then the pdf of $j^{\text {th }}$ order statistic denoted by $f_{j: n}(x)$ is given by

$$
f_{j: n}(x)=\frac{n !}{(j-1) !(n-j) !} f(x) F(x)^{j-1}(1-F(x))^{n-j}
$$


Then probability density function of the $j^{t h}$ order statistic for the HELBE distribution is given as

$$
\begin{aligned}
& f_{j: n}(x) \quad=\frac{n !}{(j-1) !(n-j) !} \frac{x \delta \delta^{\frac{1}{k}} e^{-\frac{x}{\eta}}\left(1-(1-\delta)\left(\frac{x}{\eta}+1\right)^{k} e^{-\frac{k x}{\eta}}\right)^{-\frac{1}{k}-1}}{\eta^{2}} \\
& \times\left[1-\left(\frac{\delta\left(\frac{x}{\eta}+1\right)^{k} e^{-\frac{k x}{\eta}}}{1-(1-\delta)\left(\frac{x}{\eta}+1\right)^{k} e^{-\frac{k x}{\eta}}}\right)^{1 / k}\right]^{j-1}\left[\left(\frac{\delta\left(\frac{x}{\eta}+1\right)^{k} e^{-\frac{k x}{\eta}}}{1-(1-\delta)\left(\frac{x}{\eta}+1\right)^{k} e^{-\frac{k x}{\eta}}}\right)^{1 / k}\right]^{n-j}
\end{aligned}
$$

The HELBE distribution has minimum order statistic is given by

$$
f_{1: n}(x)=\frac{x \delta^{1 / k} e^{-\frac{x}{\eta}}\left(1-(1-\delta)\left(\frac{x}{\eta}+1\right)^{k} e^{-\frac{k x}{\eta}}\right)^{-\frac{1}{k}-1}}{\eta^{2}}\left[\left(\frac{\delta\left(\frac{x}{\eta}+1\right)^{k} e^{-\frac{k x}{\eta}}}{1-(1-\delta)\left(\frac{x}{\eta}+1\right)^{k} e^{-\frac{k x}{\eta}}}\right)^{1 / k}\right]^{n-1}
$$

and the maximum order statistic is given by

$$
f_{n: n}(x)=n \frac{x \delta^{1 / k} e^{-\frac{x}{\eta}\left(1-(1-\delta)\left(\frac{x}{\eta}+1\right)^{k} e^{-\frac{k x}{\eta}}\right)^{-\frac{1}{k}-1}}}{\eta^{2}}\left[1-\left(\frac{\delta\left(\frac{x}{\eta}+1\right)^{k} e^{-\frac{k x}{\eta}}}{1-(1-\delta)\left(\frac{x}{\eta}+1\right)^{k} e^{-\frac{k x}{\eta}}}\right)^{1 / k}\right]^{n-1}
$$

\section{Data Analysis}

Here we consider the applications to three real data sets to illustrate the importance of the HELBE distribution. The goodness of fit statistics for this model is compared with competing models. The first data set is used to compare with Harris extended Lindley (HEL) distribution by Gauss M. Cordeiro et. al(2019) and Harris extended Burr XII(HEBXII) distribution by Jose and Remya (2014), the second data set is used to compare with Harris Extended Exponential(HEE) distribution by Luis Pinho et.al(2015) and the third data set is used to compare with Harris extended Lindley (HEL) distribution and Harris extended Exponentiated Exponential(HEEE) by Jose and Remya (2014). To compare the goodness of fit test using information criteria, we use Akaike Information Criteria(AIC) $=-2 \log L+2 k$ and Bayesian Information Criteria $(\mathrm{BIC})=-2 \log \mathrm{L}+\mathrm{k} \operatorname{logn}$, where $\mathrm{k}$ is the number of parameters and $\mathrm{n}$ is the sample size. The distribution with smallest values for these statistics can be chosen as the best model to fit the data.

Data set I: This data is taken from Choulakian and Stephens (2001) and it represents 72 exceedances (rounded to one decimal place) of flood peaks(in $\mathrm{m}^{3} / \mathrm{s}$ ) of the Wheaton River near Car cross in Yukon Territory,Canada for the years 1958-1984. The data is,

I. flood data

$1.7,2.2,14.4,1.1,0.4,20.6,5.3,0.7,1.9,13.0$, $12.0,9.3,1.4,18.7,8.5,25.5,11.6,14.1,22.1,1.1,2.5,14.4$, $1.7,37.6,0.6,2.2,39.0,0.3,15.0,11.0,7.3,22.9,1.7,0.1$, $1.1,0.6,9.0,1.7,7.0,20.1,0.4,2.8,14.1,9.9,10.4,10.7$, $30.0,3.6,5.6,30.8,13.3,4.2,25.5,3.4,11.9,21.5,27.6,36.4$, $2.7,64.0,1.5,2.5,27.4,1.0,27.1,20.2,16.8,5.3,9.7,27.5$, 2.5, 27.0.

To compare the goodness of fit test for HELBE, HEL and HEBXII distributios, we estimate the unknown parameters of the distributions by the method of maximum likelihood estimation. The maximum likelihood estimates,Standard error(SE), $-2 \log L$, AIC and BIC values are given in Table 1. From Table 1, it is seen that the smallest AIC, BIC values are obtained for HELBE distribution. So, we conclude that HELBE distribution is a better model to fit this data.

Table 1: Estimates and some goodness of fit measures for Data set I

\begin{tabular}{|c|c|c|c|c|c|c|}
\hline Distribution & Parameter & Estimates & SE & $-2 \log L$ & AIC & BIC \\
\hline \multirow{5}{*}{ HELBE } & $\hat{\eta}$ & 8.8200 & 1.0685 & 494.80 & 500.80 & 507.63 \\
\cline { 2 - 4 } & $\hat{\delta}$ & 0.0225 & 0.0135 & & & \\
\cline { 2 - 5 } & $\hat{k}$ & 8.8472 & 2.6334 & & & \\
\hline \multirow{3}{*}{ HEL } & $\hat{\eta}$ & 0.1100 & 0.0141 & 497.19 & 503.19 & 510.02 \\
& $\hat{\delta}$ & 0.0768 & 0.0379 & & & \\
\cline { 2 - 4 } & $\hat{k}$ & 6.1432 & 2.0361 & & & \\
\hline
\end{tabular}


"Harris Extended Length Biased Exponential Distribution and its Applications"

\begin{tabular}{|c|c|c|c|c|c|c|}
\hline \multirow{3}{*}{ HEBXII } & $\hat{\eta}$ & 0.1000 & 0.0124 & 612.85 & \multirow{2}{*}{618.85} & 627.96 \\
\cline { 2 - 4 } & $\hat{\delta}$ & 0.0673 & 0.0239 & & & \\
\cline { 2 - 4 } & $\hat{k}$ & 0.6144 & 0.2147 & & & \\
\cline { 2 - 4 } & $\hat{b}$ & 0.0999 & 0.0482 & & & \\
\hline
\end{tabular}

Data set II: This data is taken from Obubu Maxwell et.al. (2019), consisting of 100 observations on tensile strength of carbon fibers. The data is,

II. Tensile strength of carbon fibre.

$$
3.7,3.11,4.42,3.28,3.75,2.96,3.39,3.31,3.15 \text {, }
$$
$2.81,1.41,2.76,3.19,1.59,2.17,3.51,0.84,1.61,1.57,1.89$, $2.74,3.27,2.41,3.09,2.43,2.53,2.81,3.31,2.35,2.77,2.68$, $4.91,1.57,2.00,1.17,2.17,0.39,2.79,1.08,2.88,2.73,2.87$, $3.19,1.87,2.95,2.67,4.20,2.85,2.55,2.17,2.97,3.68,0.81$, $1.22,5.08,1.69,3.68,4.70,2.03,2.82,2.50,1.47,3.22,3.15$,
2.97, 2.93, 3.33, 2.56, 2.59, 2.83, 1.36, 1.84, 5.56, 1.12, 2.48, $1.25,2.48,2.03,1.61,2.05,3.60,3.11,1.69,4.90,3.39,3.22$, $2.55,3.56,2.38,1.92,0.98,1.59,1.73,1.71,1.18,4.38,0.85$, $1.80,2.12,3.65$.

The maximum likelihood estimates,Standard error(SE),-2log $L$, AIC and BIC values are given in Table 2. From Table 2, it is seen that the smallest AIC, BIC values are obtained for HELBE distribution. So, we can conclude that HELBE distribution is a better model to fit this data.

Table 2: Estimates and some goodness of fit measures for Data set II

\begin{tabular}{|c|c|c|c|c|c|c|}
\hline Distribution & Parameter & Estimates & SE & -2logL & AIC & BIC \\
\hline \multirow{2}{*}{ HELBE } & $\hat{\eta}$ & 0.5270 & 0.1247 & 284.31 & 290.31 & 298.13 \\
\cline { 2 - 5 } & $\hat{\delta}$ & 21.8769 & 9.6823 & & & \\
\cline { 2 - 5 } & $\hat{k}$ & 1.0115 & 0.4633 & & & \\
\hline \multirow{2}{*}{ HEE } & $\hat{\lambda}$ & 1.6693 & 0.4383 & 285.25 & 291.25 & 29.06 \\
\cline { 2 - 4 } & $\hat{\theta}$ & 71.3542 & 32.1561 & & \\
\cline { 2 - 5 } & $\hat{k}$ & 0.9959 & 0.4050 & & & \\
\hline
\end{tabular}

Data set III: This data is taken from Lawless (2003), it represents the failure times (in minutes)for a sample of 15 electronic components in an accelerated life test. The data is,

III. The failure times

$1.4,5.1,6.3,10.8,12.1,18.5,19.7,22.2,23,30.6$
37.3, 46.3, 53.9, 59.8, 66.2.

The maximum likelihood estimates, AIC and BIC values, and $\mathrm{K}-\mathrm{S}$ statistic are given in Table 3. From Table 3, it is seen that the smallest AIC, BIC values are obtained for HELBE distribution. So, we conclude that HELBE distribution is a better model to fit this data.

Table 3: Estimates and some goodness of fit measures for Data set III

\begin{tabular}{|c|c|c|c|c|c|c|}
\hline Distribution & Parameter & Estimates & SE & $-2 \log \mathrm{L}$ & AIC & $\mathrm{BIC}$ \\
\hline \multirow[t]{3}{*}{ HELBE } & $\hat{\eta}$ & 15.0982 & 3.3243 & \multirow[t]{3}{*}{127.86} & \multirow[t]{3}{*}{133.86} & \multirow[t]{3}{*}{135.98} \\
\hline & $\hat{\delta}$ & 0.1225 & 0.2410 & & & \\
\hline & $\widehat{k}$ & 20.3251 & 35.4556 & & & \\
\hline \multirow[t]{3}{*}{ HEL } & $\hat{\eta}$ & 12.7036 & 27.0292 & \multirow[t]{3}{*}{128.04} & \multirow[t]{3}{*}{134.05} & \multirow[t]{3}{*}{136.18} \\
\hline & $\hat{\delta}$ & 0.0648 & 0.0146 & & & \\
\hline & $\widehat{k}$ & 0.2959 & 0.4875 & & & \\
\hline \multirow[t]{4}{*}{ HEEE } & $\hat{\eta}$ & 0.2147 & 0.1214 & \multirow[t]{4}{*}{499.82} & \multirow[t]{4}{*}{505.82} & \multirow[t]{4}{*}{508.65} \\
\hline & $\hat{\delta}$ & 2.2640 & 0.8353 & & & \\
\hline & $\hat{k}$ & 0.3661 & 0.1127 & & & \\
\hline & $\hat{\alpha}$ & 0.1629 & 0.0230 & & & \\
\hline
\end{tabular}

\section{Conclusion}

The HELBE distribution has been successfully introduced in this paper and some of its statistical properties including asymptotic properties, the maximum likelihood estimation of the model parameters and density function of the order statistic have been obtained. The density function of the model and its failure rate have unimodal shapes; this means that the model would be useful to fit real life events with unimodal failure rates. The real data sets are used to illustrate the flexibility and compatibility of the new distribution and compare the results with an existing distributions. Thus, this newly developed distribution can serve as a complementary model in lifetime modelling as well as reliability studies. This distribution has so many applications in diverse fields. In future, some more properties and applications of this 
distribution can be explored and reliability test plans can also be conducted.

\section{References}

1. Aly, E.A.A., and Benkherouf, L. (2011). A new family of distributions based on probability generating functions. Sankhya $B$, 73, 70-80.

2. Batsidis, A., and Lemonte, A.J.(2014). On the Harris Extended Family of Distributions. Statistics: A Journal of Theoretical and Applied Statistics,49(5) ,1400-1421.

3. Choulakian, V., and Stephens, M.A. (2001). Goodness of fit for the generalized Pareto distribution. Technometrics, 43, 478-484.

4. Dara, S.T., Ahmad, M.(2012). Recent Advances in Moment Distribution and Their Hazard Rates. Lap Lambert Academic Publishing GmbH KG.

5. Gauss M. Cordeiro1, M. Mansoor, and Serge B. Provost(2019). The Harris extended Lindley distribution for modeling hydrological data. Chilean Journal of Statistics ,10(1), 77-94.

6. Harris, T.E.(1948). Branching Processes. Ann Math Stat. 19,474-494.

7. Jose, K.K., Remya, S.(2014). Harris Extended Burr XII and Exponentiated Exponential Distribution. Pre-Print.

8. Jose, K.K. and Paul, A. (2018). Reliability Test
Plans for Percentiles Based on the Harris Generalized Linear Exponential Distribution. Stochastics and Quality Control (online: https://doi.org/10.1515/eqc-2017-0025).

9. Jose, K.K., Lishamol Tomy and Sophia P. Thomas.(2018). On a Generalization of the Weibull Distribution and Its Application in Quality Control. Stochastics and Quality Control, 33(2), 113-124.

10. Jose, K.K. and Varghese J.(2019).Harris Extended Log-Kumaraswamy Distribution And Its Applications. IAPQR Transaction 44(1),20-37.

11. Lawless, J. F.(2003). Statistical models and methods for lifetime data. Wiley, New York.

12. Muhammad, A.H.,Rana, M.U.,Sharqa, H. and Amer, I.A.(2017).The Marshall-Olkin length-biased exponential distribution and its applications.Journal of King Saud University,31 , 246-251.

13. Obubu Maxwell, Samuel Oluwafemi Oyamakin, Angela Unna Chukwu, Yusuf Olufemi Olusola, Adeleke Akinrinade Kayode.(2019). New Generalization of Length Biased Exponential Distribution with Applications. Journal of Advances in Applied Mathematics, 4 (2).

14. Pinho, L.G.B., Cordeiro, G.M., Nobre, J.S.(2015). The Harris Extended Exponential Distribution. Commun Stat Theory Methods, 44 ,3486-3502. 